\title{
TECNOLOGÍA APLICADA EN EL FUNCIONAMIENTO Y LA DETECCIÓN DE MINAS ANTIPERSONALES: ESTADO DEL ARTE
}

\section{Technology applied in the operation and detection of antipersonnel mines: state of the art}

\author{
Javier Andrés Ledezma-Ríos \\ Ing. Universidad del Cauca (Popayán, Cauca, Colombia). jaledezma@unicauca.edu.co
}

(Recibido junio 21 de 2016 y Aprobado junio 2 de 2017)

\begin{abstract}
Resumen
El principal objetivo de esta investigación es conocer las diferentes tecnologías implementadas para la detección de minas antipersonales. Por diferentes medios bibliográficos se estudiaron las últimas actualizaciones empleadas para la detección de objetos enterrados, los factores que afectan la pérdida de energía de las ondas como transmisoras de información entre estos, las características del suelo, la amplitud de la señal emitida, la frecuencia y las condiciones del terreno. En este artículo se informa sobre los medios computacionales, de su trabajo con los diferentes algoritmos para modelar una información acertada de lo que está sucediendo con el fenómeno de detección. Asimismo, se dan a conocer a la comunidad científica los parámetros de susceptibilidad magnética, el porcentaje de agua y porosidad del entorno donde reaccionan las ondas emitidas, la dificultad de la estabilidad de la señal que se ha de capturar para detectar las minas antipersonales, en un contorno geográfico. En la actualidad se están utilizando tubos de PVC, latas y jeringas para su fabricación, y dispositivos de manipulación manual para su activación. Las ondas van a tener un comportamiento diferente ante estos materiales.
\end{abstract}

Palabras clave: investigación; ondas; terreno; transmisoras; fabricación.

\begin{abstract}
The main objective of this investigation is to know the different technologies implemented for the detection of antipersonnel mines, documented by different bibliographic means of the latest updates used for the detection of buried objects, the factors that affect the loss of energy of the waves as transmitters of information between them, the characteristics of the soil, the amplitude of the emitted signal, the frequency and the conditions of the terrain. This paper informs about the computational means, of their work with the different algorithms to model correct information of what is happening with the phenomenon of detection. Thus, through this research, the scientific community is informed on the parameters of magnetic susceptibility, the percentage of water and porosity of the environment where the emitted waves react, the difficulty of the stability of the signal to be captured to detect antipersonnel mines, in a geographical context. Currently, PVC tubes, cans, syringes and hand-held devices are being used for their production, and the waves will behave differently against these materials.
\end{abstract}

Keywords: research; waves; terrain; transmitter; manufacturing.

\section{INTRODUCCIÓN}

Las ondas son fenómenos físicos que tienen la capacidad de llevar y traer información, lo que ha permitido trabajar en muchas aplicaciones en cuanto a la transmisión de datos en diferentes campos de estudio [1], como es el caso de las telecomunicaciones, la radio y el radar. Las señales emitidas capturadas y convertidas, en muchos casos, nos dan una idea de comportamientos de la naturaleza, de ahí que el ser humano reconoce la importancia de su estudio. Por esto se generan proyectos de investigación enfocados en las ondas y la detección de las minas terrestres. Es importante destacar que algunos autores definen estos artefactos como explosivos improvisados [2]. Una de las formas de transmisión y recepción posibles para ser utilizadas como detecto-

Cómo citar este artículo:

J.A. Ledezma-Rios, "Tecnología aplicada en el funcionamiento y la detección de minas antipersonales: estado del tema", Revista Ingeniería Investigación y Desarrollo, vol. 17 (2), pp. 23-35, Julio, 2017. 
res de objetos enterrados, bajo el concepto de radar de penetración terrestre (GPR) [3], es la tecnología que utiliza ondas electromagnéticas para detectar objetos bajo la superficie de la tierra y mapear suelos y zonas subterráneas [1]. En este caso, la ubicación y las propiedades de los objetos enterrados son determinadas por los datos de dispersión.

Este método es el más utilizado en función del objeto y de las ecuaciones integrales de dispersión de ondas. $\mathrm{Si}$ las soluciones son lo suficientemente precisas, entonces sus características de localización, forma y reflexión pueden ser determinadas y, por lo tanto, el objeto puede ser identificado. La onda emitida desde el radar se puede aproximar a una señal continua oscilante que puede expresarse como una función de coseno desfasada o en fase. La frecuencia, el tiempo y la fase de la onda que incide en el suelo, nos permiten analizar la presencia de algún objeto enterrado, debido a las características de la onda que es reflejada y que viajará en dirección opuesta al incidente. Una vez en el punto donde se genera la señal, es posible expresar la onda reflejada mediante una ecuación dependiente de la distancia desde la fuente hasta el objeto enterrado y de la velocidad de propagación de las ondas en el medio. Finalmente, la amplitud de la señal de interferencia [4], a partir de la cual se conforman las imágenes, queda dependiendo de la distancia, la frecuencia y la velocidad de propagación, mas no del tiempo ni de la fase de las señales.

En este punto cabe mencionar algunos trabajos recientes en las imágenes de GPR obtenidas del suelo en efectos de difracción y controladas mediante algoritmos de programación, y mostradas mediante graficas de diseño computacional, a partir de datos obtenidos por el radar de penetración en la tierra, mono o múltiestático de multifrecuencia.

El acompañamiento de un algoritmo basado en la aproximación de Born para el vector de dispersión y condiciones electromagnéticas que incorpora modelos neareld es una tecnología de comunicación inalámbrica de corto alcance y alta frecuencia que permite el intercambio de datos entre dispositivos reales para la recepción y transmisión. El modelo de dispersión hacia adelante se invierte analíticamente usando el operador de la pseudoinversa, regularizada para obtener imágenes de la región subterránea, basándose en mediciones dispersas de un receptor. Mientras que los algoritmos de inversión habituales de DT, nombre definido para este tipo de algoritmo, requieren un medio y un fondo para que no haya pérdidas de fuentes puntuales [5] y receptores ideales, otro algoritmo diseñado por Deming y de Devaney permite un fondo atenuante y una transmisión arbitraria de los receptores.

\section{METOdOLOGÍA}

En estas secciones van, entre otras herramientas metodológicas, procesos experimentales, técnicas de diseño previo, revisión bibliográfica, últimas investigaciones de radar de penetración terrestre, técnicas de recolección de datos, tablas de información y diferentes recursos proporcionados por los autores.

\section{1 Últimas investigaciones del radar de penetración} En las últimas décadas, la llegada del radar de penetración (GPR) ha dado lugar a la revolución multidisciplinar en el campo de la detección de objetos enterrados, con una amplia aplicación en áreas como la arqueología [6], la geología [7] y la industria militar (la detección de minas) [8]. Uno de los principales retos en los sistemas GPR, más allá de la mera detección de objetos enterrados, es reunir información sobre la composición de los objetos o el medio ambiente que los rodea. Aunque la tecnología electrónica necesaria para llevar a cabo esos sistemas ahora madura con la evolución constante [5,6], persisten limitaciones en la detección e interpretación de los resultados previstos. En los últimos años dos líneas principales han surgido para resolver este problema. Por un lado, se aplican algunos sistemas de técnicas gráficas tomografía $[9,10]$, así como aplicaciones usando ecuaciones de las integrales[11] , pero estos han tenido un éxito solo parcial, debido principalmente a la complejidad de los datos de campo, que contiene altos niveles de ruido causados por no homogeneizar los medios de acogida. Por otro lado, las técnicas basadas en redes neuronales (NN) que aprenden a adaptarse basándose en las experiencias recogidas en diferentes topologías [11-17] conocidas como neural network, se han propuesto para resolver el problema del electromagnetismo y la inversión canónica. Por ejemplo, un esferoide incrustado en un medio de acogida $[17,18]$, y otras mejoras en relación con las formas geométricas más realistas para aplicaciones ingenieriles $[18,19]$, aun incluyendo la consideración de un medio de acogida no homogénea $[19,20]$. Un punto común en todas estas redes neuronales (NN), es la aplicación como paso previo 
a la fase de entrenamiento de las neural network, de un modelo computacional de escenarios de GPR.

De esta manera, el campo dispersado en un escenario generado al azar se puede calcular por métodos numéricos, por lo general en finitas diferencias en el dominio del tiempo (FDTD), un ventaneo en el dominio de la frecuencia $[17,18]$, o para los casos en que se presentan inestabilidades numéricas, la dirección alterna de la FDTD implícita (ADI-FDTD) es un método $[19,20]$ de aplicación. Una de las principales deficiencias de la aplicación de las NN como un sistema de predicción de problemas GPR es la maldición de la dimensional dad, que hace que el entrenamiento lento y la capacidad de predicción del sistema sea más pobre [21-24].

Por lo tanto, un punto clave es reducir la alta dimensionalidad de los datos de campo dispersos, lo que permite un número reducido de entradas, para lo cual se capacitó a las NN, haciendo el proceso más rápido y fiable. En este punto, la aplicación de la técnica de procesamiento de señal, que es como el análisis de componentes principales (PCA), puede ser introducida como parte del algoritmo. La utilidad de PCA como una técnica de compresión con pérdida mínima de información en señales de GPR en el dominio del tiempo se ha demostrado en $[19,20]$. En este trabajo, el objetivo es estimar la profundidad y el radio de tubos enterrados en una estructura de hormigón no homogénea. En el contexto del presente trabajo se pretende aplicar técnicas basadas en la PCA y NN, a fin de construir sistemas de predicción para las características geológicas, basados en GPR. Por otra parte, este procedimiento principal no solo es para lograr una tasa alta de éxito en las predicciones, sino también para construir trabajos previos para esta línea de investigación, mediante la producción de resultados y gráficos B-scan. En este sentido, el algoritmo propuesto supera las anteriores predicciones de las $\mathrm{NN}$, al proporcionar salidas numéricas de una sola dimensión, pues es lo que permite la interpretación de las soluciones de los usuarios no especializados en el procesamiento de datos GPR.

En primer lugar, se proporciona una visión general de la teoría de fondo, describiendo brevemente el algoritmo PCA, el NN de las redes neuronales y la creación de datos sintéticos con FDTD. Se presenta el esquema del sistema de predicción, con especial atención en las diferencias en la aplicación de las encuestas A y B-scan. Otra sección muestra la influencia de algunos parámetros nu- méricos en el rendimiento del sistema de predicción y, finalmente, se proporcionan ejemplos ilustrativos relacionados con la detección y predicción de las capas geológicas. Lo más interesante de este artículo es la forma y utilización del entrenamiento de las redes neuronales para el procesamiento digital de la señal, que puede servir en un trabajo futuro dentro de investigación en el desarrollo de la red neuronal que se debe entrenar para obtener el mejor patrón de la señal de las ondas en nuestro caso.

\subsection{Redes neuronales (NN)}

La Figura 1 muestra el flow chart que sistematiza el sistema de predicción. El algoritmo de predicción se puede describir como un sistema modular que combina tres diferentes recursos en el proceso: (1) códigos numéricos de simulación electromagnética, (2) técnicas de compresión de procesamiento de señales, y (3) la teoría de redes neuronales. Otras mejoras y avances de entrada en cualquiera de estas teorías se podrían acomodar en cada etapa del proceso.

El primer paso en el desarrollo de un algoritmo de predicción basado en redes neuronales NN es reunir datos representativos de situaciones en las que la red neuronal va a funcionar. La adecuada realización de la formación y configuración de fases NN estará directamente relacionada con la diversidad, cantidad y calidad de los datos proporcionados. Para los sistemas GPR, el uso de los datos experimentales es difícilmente asequible debido a que (1) requiere mucho tiempo y mano de obra intensiva, y (2) apenas es libre de objetos no deseados y otras fuentes [5] experimentales de errores. Por estas razones, se considera el uso de los datos de entrada a partir de simulaciones electromagnéticas.

El enfoque numérico FDTD para las soluciones de las ecuaciones de Maxwell se ha empleado ampliamente para simulaciones GPR $[21,22]$. Este proporciona una mayor precisión a los métodos de trazado de rayos [- $T$ T 25], pero aumenta la carga computacional. Por otra parte, los escenarios realistas GPR pueden resolverse debido a la capacidad de tratar con materiales no homogéneos y dispersivos. Sin embargo, en algunos casos, las inestabilidades numéricas pueden surgir, invalidando los resultados calculados. En tal caso, se acude a una versión mejorada de la parte numérica de la FDTD, llamada ADI-FDTD, que se basa en una formulación secuencial implícita finita de Maxwell. 


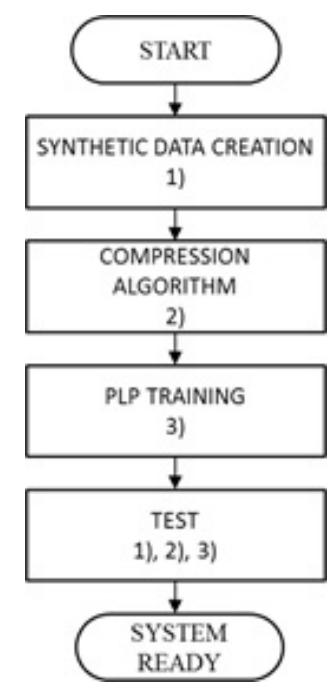

Figura 1. Diagrama de flujo del sistema de predicción. Los nú meros significan los recursos externos aplicados en cada pasō [26].

Las ecuaciones en el dominio de tiempo pueden generar resultados precisos. Por lo tanto, el modelado adecuado del equipo GPR y diferentes escenarios posibles (por ejemplo, fuentes electromagnéticas, alimentación del pulso, parámetros de materiales constitutivos, geometrías de suelos no homogéneos) pueden ser eficientemente introducidos con la ayuda de secuencias de comandos. Se requiere que la automatización de procesos de la fase de entrenamiento sea típicamente para funcionar cientos de casos de la red NN, donde se pueda determinar, para luego ser nuevamente entrenada. Las técnicas de compresión de señales de procesamiento han constituido un campo activo de investigación en las últimas décadas, principalmente para aplicaciones relacionadas con el procesamiento de audio e imagen [24-26], que sería otra forma de procesar la información. Fueron diseñadas inicialmente para los sistemas de comunicación que están destinados a manejar una gran cantidad de información con la menor cantidad de datos posibles.

En este sentido, el problema considerado aquí es análogo a la situación de la enorme cantidad de datos obtenidos a partir de GPR por simulaciones electromagnéticas, lo que hace que su uso directo sea ineficiente para la figuración de la NN, debido principalmente a la alta complejidad de los algoritmos de entrenamiento, que requieren la manipulación de los datos de entrada de cientos de simulaciones con el fin de determinar las variables y NN pesos. Incluso en escenarios en los que un coeficiente elevado de número de simulaciones se puede calcular, es posible que los algoritmos avanzados de formación no converjan para alta dimensiones NN, debido principalmente a la dificultad de proporcionar un conjunto no escasa de datos de entrenamiento $[24,25]$. Por esta razón, se hace necesario para procesar los datos sintéticos y eliminar la información redundante. Esta redundancia de datos es una característica negativa típica de los sistemas GPR, donde las mediciones exhaustivas se realizan en el mismo escenario y aparecen diferencias menores entre las trazas adyacentes. Para explotar la fuerte correlación en los datos, se puede aplicar el análisis de componentes principales (PCA) [27-31]. PCA identifica patrones similares en los datos y reorganiza los datos de tal manera que las similitudes y diferencias se resaltan. Matemáticamente, esto se consigue con una ortogonalización de una matriz construida mediante la adición de filas con trazas de datos de entrada, de modo que estas filas no estén relacionadas entre sí. Otra característica principal de la PCA es que una vez que se encuentran estos patrones en los datos (por ejemplo, se determina la base ortogonal), estos últimos también se pueden comprimir sin pérdida significativa del peralte de la información, por la simple eliminación de algunos de los vectores de la base. En el presente trabajo, la capacidad de compresión de la PCA se utiliza para extraer la información más relevante del conjunto de datos empleado en la fase de entrenamiento NN. La designación de los componentes principales se hace desde una traza conociendo los componentes de la nueva base ortogonal que se deriva de los datos de la matriz inicial. Con una reducción en la dimensión de la base se puede realizar un bajo impacto sobre la traza original y el número de componentes principales se puede reducir, lo que conduce a bajas dimensiones NN.

La siguiente sección mostrará que la descomposición de diferentes señales $[28,29]$ GPR utilizando el PCA es un factor clave de la capacidad de NN para reconstruir los escenarios geológicos originales. Además, son necesarias algunas consideraciones relativas a la NN al final de la descripción de las teorías sobre las que se fundamenta el sistema propuesto. El desarrollo inicial de la base teórica de los sistemas de NN [27-29] se ha dado en los últimos años, seguido de un periodo de madurez de aplicaciones del mundo real [30-32]. Una decisión importante en cualquier problema por resolver es la elección de la topología de red, ya que esta topología tiene un impacto significativo en el rendimiento del sistema y en el mismo problema, que a menudo se puede 
resolver con éxito con diferentes topologías. Entre un número considerable de topologías de red [33,34], el trabajo descrito aquí ha utilizado una topología particular llamada Paralelo-Layer Perceptrón (PLP) [25,11]. En cuanto al problema de inversión, esta topología tiene algunas ventajas sobre otras redes clásicas, tales como la multicapa de percepción (MLP) y el sistema basado en la red de adaptación fuzzy-inferencia (ANFIS) [35-40].

Además, el PLP ofrece mejor rendimiento que el MLP, manteniendo al mismo tiempo la capacidad de ANFIS para manejar problemas com plejos. El algoritmo de entrenamiento para el PLP se usa en el presente documento con un método híbrido basado en el algoritmo de aprendizaje de propagación hacia atrás [37-40], que combina una estimación por mínimos cuadrados (LSE) y la corrección de segundo orden algoritmo de Levenberg-Maquardt [38-46]. Los estudios preliminares con este algoritmo de entrenamiento han dado resultados satisfactorios en términos de tiempo de entrenamiento y de precisión de las soluciones, y también ha mostrado un rendimiento superior en comparación con los algoritmos alternativos, tales como el algoritmo de descenso de gradiente y un descenso de gradiente híbrido y método de LSE.

\subsection{Herramienta NN-PCA para la detección basada en GPR}

El algoritmo desarrollado en este proyecto tiene como objetivo la predicción de la composición y estructura en escenarios complejos GPR, donde pueden aparecer múltiples homogeneidades. De esta manera, los recintos simulados emulan hosts físicos en la medida de lo posible, lo que facilita llevar a cabo nuevas investigaciones sobre las mediciones experimentales. Con este fin se hace una malla cartesiana para el escenario que se está probando, y el sistema propuesto proporciona un mapa de salida del medidor apartado, constitutivo de cada cuadrícula. Mediante la transformación de estos valores, el software puede estimar un material físico para cada parte de la malla, reconstruyendo de ese modo la imagen original GPR, en la que cada píxel corresponde a una caja de la malla cartesiana.

En esta sección se presenta una descripción detallada para el modelo 1D. Este enfoque se denomina 1D, porque se basa solo en GPR A-scan, es decir, solo un rastro de cada sección se considera para las etapas de formación y de predicción. A continuación, una subsección está dedicada a la explicación de una extensión de la 1D-modelo anterior para considerar B-scan más frecuentes en las encuestas experimentales GPR; lo que resulta en un algoritmo mejorado llamado 2D-modelo.

2.3.1 1D-modelo. La Figura 2 muestra gráficamente el proceso para generar un PLP-NN basado en trazas A-escaneo para fines de predicción. Se indica el proceso de entrenamiento de la red neural con los bloques de redes neuronales y la capa de perceptrón paralelo, que se divide en simulación computacional (a) y formación de la matriz (b). Las principales etapas del algoritmo se describen a continuación:

2.3.1.1 Determinación de la matriz de capacitación. La primera etapa consiste en una generación aleatoria de escenarios mallados (es decir, diferentes permitividades en cada bloque), que son simulados por medio de ADIFDTD. Con las tensiones medidas en la antena de recepción (considerado como un dipolo de Hertz), se toman en cada escenario como la salida de las simulaciones. Esta serie de tensiones en el tiempo se conoce como trazas (como se muestra en la Fig. 3 (a)). Todos los restos son agrupados y dispuestos para formar una matriz de datos rectangular de dimensiones número- de-ejemplos tamaño, que se señaló como (b) de la misma figura rastro. El valor del tamaño de traza se determina por el número de intervalos de tiempo que resuelve el simulador GPR electromagnético. En este punto, vale la pena mencionar que en algunos casos es útil para preprocesar los datos, eliminando de este modo la primera reflexión, debido a la planta de la interfaz de aire, que no tiene ningún interés en la predicción de los materiales enterrados. En la práctica, esto se consigue restando la traza correspondiente a un escenario compuesto exclusivamente por el anfitrión, sin materiales adicionales en el interior.

2.3.1.2 Procesamiento de la matriz de datos. La matriz de datos tiene alta dimensionalidad, pero los componentes traza están estrechamente correlacionados porque corresponden a exploraciones electromagnéticas sucesivas de escenarios geológicos que tienen diferentes reflexiones en características de diferentes permitividades (es decir, como centros de dispersión) incrustadas en el mismo host medio. En este caso, la aplicación del algoritmo PCA reduce la alta dimensionalidad de los datos de la matriz. Esta reducción es un paso clave en el rendimiento del sistema de predicción, porque el uso de altas dimensiones, por ejemplo, los NN, NN, que contienen un elevado número de entradas/salidas para hacer 
frente a la información proporcionada por los campos dispersos, darían lugar a la ineficacia de los sistemas de predicción, incluso en los casos en que la etapa de formación de la NN se puede lograr. PCA extrae las principales características de las huellas, por medio de una descomposición ortogonal de los vectores de entrada, por ejemplo, filas de los datos de la matriz en un conjunto de huellas no físicas, agrupados en una nueva matriz de dimensiones número de PCA tamaño traza, como el nombre de la matriz de señal. La proyección matemática de GPR-huellas en la matriz de la señal dará lugar a los principales componentes de estos restos, que se corresponden con el peso de cada señal de PCA para reconstruir la traza inicial. Estos pueden estar dispuestos formando una serie de ejemplos número de matriz de PCA, que se llama como la matriz de PCA. Matemáticamente, esta operación se expresa como:

\section{DATA Matrix $=($ PCAX Matrix Signal Matrix $)(1)$}

Procedimientos numéricos conocidos para el cálculo de la matriz de señales se pueden encontrar en $[29,30]$. Sin embargo, vale la pena señalar los fundamentos de la reducción de la dimensionalidad del NN. Con este fin, un factor clave es el significado del número de parámetro de la ACP. Cuanto mayor sea este valor, mayor será la precisión en la descomposición de la traza original en la nueva base de vectores. Sin embargo, no todos los vectores de la nueva base contribuyen de la misma manera a la descomposición. PCA no solo determina la matriz de señal, sino también da una medida de la información contenida en cada fila de esta matriz, para la reconstrucción de la matriz de datos, atendiendo a los valores numéricos que componen la matriz de PCA. Por lo tanto, es posible eliminar de forma selectiva aquellas filas con menos contribución de la información al conjunto de datos original, actuando así como una compresión de la matriz de datos. Si se tiene en cuenta que el número de parámetro PCA corresponde al número de las neuronas en la capa de entrada, se hace evidente que hay ventajas computacionales derivadas de una reducción efectiva de la nueva base de vectores, que afecta solo a esas señales pero si hay pocas neuronas en la capa de entrada la información no se puede obtener completa porque no hay una disminución de los vectores de la base y esto no ayuda a predecir el comportamiento final de la información de la señal. Por otra parte, la matriz de señal juega un papel importante en la etapa de predicción. Una vez que se calcula la matriz de señal comprimida, se puede determinar el conjunto de componentes de PCA correspondiente a la nueva traza. Así, en este artículo se ve la importancia del procesamiento de señales y su aplicación. En la siguiente sección veremos el estudio en el tema de minas y explosivos.

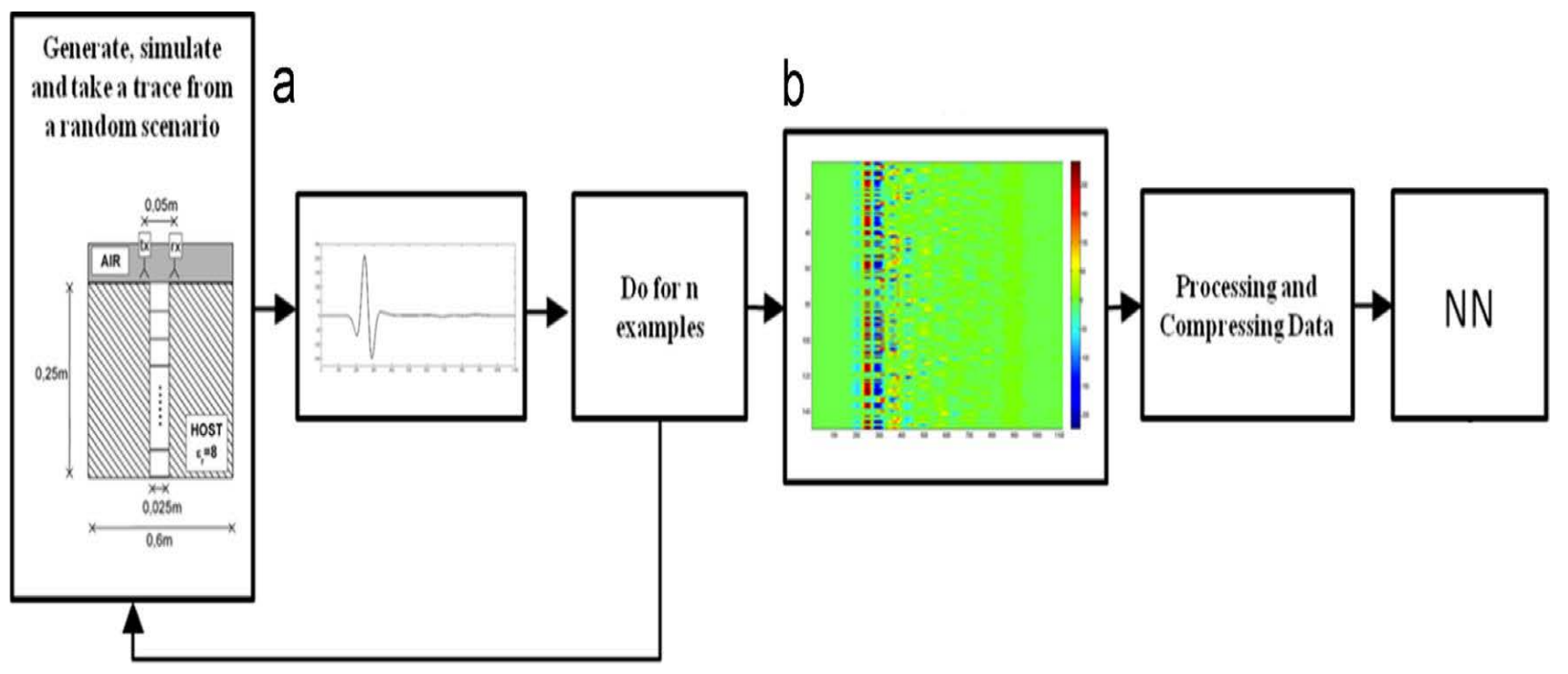

Figura 2. Esquema ilustrado de la simulación computacional de un solo escenario (a) y la formación de la matriz de datos (b) [26].

Así, el aporte de esta información será en la realización en la parte de la programación de las redes neuronales, mediante un algoritmo que puede ser tenido en cuenta para el mejor aprovechamiento de la información obtenida dentro de la matriz de datos para ser procesada. 


\subsection{Las características del suelo de las minas}

Hay dos tipos de suelos afectados por las minas en Colombia: suelo franco limo y arena arcillosa, respectivamente; su contenido orgánico es inferior al $1 \%$ y su color rojizo se debe a la presencia de óxidos mixtos que contienen partículas de hierro finas (su porcentaje Fe es $5,2 \%$ y $8,8 \%$, respectivamente). Estos suelos hacen que la detección de minas AP usando minidiscos sea más complicada [42], debido a su alta susceptibilidad magnética ( $M S, \chi$ ) y a la viscosidad magnética (o MS dependientes de la frecuencia, $\chi F D$ ) [43]. Según lo investigado con el fin de determinar el efecto de los componentes de hierro sobre las propiedades EM, se mide la susceptibilidad magnética de los suelos seleccionados utilizando un sensor Barington MS2 de doble frecuencia $(0,46$ / 4,6 KHz). [44] Se escogen varias muestras tomadas de la parte superior a $30 \mathrm{~cm}$, de acuerdo con la fuente de información [2]. Se obtienen dos mediciones: MS en baja frecuencia ( $\mathrm{XLF}$ ) y MS en alta frecuencia ( $\mathrm{XHF}$ ). De esta manera, el MS es dependiente de la frecuencia y se puede expresar como una pérdida relativa de la susceptibilidad (XFD $=\chi$ LF $-\chi \mathrm{HF}$ ) [41-44]. Los resultados de estas mediciones y otras propiedades disponibles de ambos suelos se resumen en la Tabla 1 [2].

Tabla 1. Propiedades disponibles de los dos suelos ferruginosos [2]

\begin{tabular}{cccccccccc}
\hline Textura & $\begin{array}{c}\text { Arena } \\
(\%)\end{array}$ & $\begin{array}{c}\text { Limo } \\
(\%)\end{array}$ & $\begin{array}{c}\text { Arcilla } \\
(\%)\end{array}$ & $\begin{array}{c}\rho S \\
(\%)\end{array}$ & $\begin{array}{c}\text { er } \\
(\%)\end{array}$ & $\begin{array}{c}\text { OS } \\
(-)\end{array}$ & $\begin{array}{c}\text { XRF } \\
(-)\end{array}$ & $\begin{array}{c}\text { XFD } \\
(-)\end{array}$ \\
\hline $\begin{array}{c}\text { Sedimentos } \\
\text { Porosos }\end{array}$ & 30.6 & 63 & 6.4 & 1.18 & 4.4 & 38.9 & 307 & 304 & 2 \\
$\begin{array}{c}\text { Arena } \\
\text { Arcillosa }\end{array}$ & 72.1 & 26.9 & 1 & 1.3 & 3.2 & 41 & 1080 & 1070 & 11 \\
\hline
\end{tabular}

2.4.1 Características previstas. El Ejército de Colombia usó minas terrestres AP para proteger las áreas de telecomunicaciones y las bases de la institución hasta 1995. Aunque estos se importan principalmente de los EE.UU. (M14) y de Bélgica (NR-409), unos pocos fueron producidos por INDUMIL (MN-MAP-1, MN-MAP-2).
Todas estas son las minas de bajo contenido metálico (algunas de sus características se resumen en la Tabla 2). Por lo general, su material es de polietileno (PE), polipropileno (PP), poliestireno (PS), caucho o baquelita, y su carga principal es una mezcla de diferentes componentes químicos (ver Tabla 2). Su $\varepsilon$ constante dieléctrica se calcula promediando de la constante dieléctrica el material de la caja y de la constante dieléctrica la principal carga, cuyos valores se toman a partir de Von Hippel (1995). Las zonas afectadas por las minas también están contaminadas con artefactos explosivos improvisados, que causan el $80 \%$ de las lesiones por minas terrestres en Colombia. Estos dispositivos no son producidos en masa, pues se fabrican en diferentes formas con el uso de AK de diferentes materiales con un mínimo o ningún contenido de metal, lo que hace que la detección de los no metálicos sea una tarea difícil. El tamaño y la forma de los IED pueden variar de un pequeño trozo de PVC $(10 \mathrm{~cm} \times$ $14 \mathrm{~cm}$ a $2 \mathrm{~cm}$ superficiales), en relación con la profundidad de enterramiento, que se mide desde la parte superior de la mina. Los valores dieléctricos son un promedio de las constantes dieléctricas del material de la caja y de la carga principal. Valores tomados de la enfermedad de von Hippel: -un polvo de aluminio atomizado $=$ AAP, $\mathrm{PETN}=$ pentaeritrita tetranitrato, $\mathrm{RDX}=$ ciclotrimetileno trinitramina, tetril = trinitrofenilo, $\mathrm{TNT}$ = trinitrotolueno.

2.4.2 Modelado del radar de penetración terrestre. Todos los fenómenos electromagnéticos, en una escala macroscópica, se describen mediante las ecuaciones de Maxwell. Estos son de primer orden ecuaciones diferenciales parciales que tienen que ser resueltas de acuerdo a la geometría de los objetos analizados por el radar.

Matemáticamente expresan la relación entre las cantidades de campos electromagnéticos fundamentales y la dependencia de sus fuentes, con el fin de simular la respuesta GPR de una diana particular o un conjunto de objetivos embebidos en el suelo [45]. 
Tabla 2. Resumen de las características de las minas. Esta tabla muestra los principales materiales que se utilizan en la fabricación de minas [2].

\begin{tabular}{cccccccc}
\hline $\begin{array}{c}\text { Objetivo } \\
-\end{array}$ & Diámetro & Altura & \multicolumn{2}{c}{$\begin{array}{c}\text { Material } \\
\text { Material }\end{array}$} \\
\hline $\begin{array}{c}\text { Sedimentos } \\
\text { Porosos }\end{array}$ & M14 & 5 & 64 & Plastico & TETRY & $0-15$ & 0.8 \\
\hline $\begin{array}{c}\text { (cm) } \\
\text { (cm) }\end{array}$ & NR409 & 8.2 & 2.8 & Plastico & $\begin{array}{c}\text { TNT+AAP } \\
\text { RDX }\end{array}$ & $0-15$ & 3.1 \\
$\begin{array}{c}\text { Arena } \\
-\end{array}$ & MN & 7.5 & 7 & Plastico & PETN+TNT & $0-15$ & 3.1 \\
\hline
\end{tabular}

Uno de los programas numéricos disponibles para el cálculo de GPR propagación de las ondas y de la señal propiedades es GprMax, que resuelve las ecuaciones de Maxwell utilizando el método de diferencias finitas en tiempo y el dominio. Las FDTD [61] son una de las técnicas que se basan en discretizar el espacio y el tiempo utilizando una retícula ortogonal y la solución de la forma diferencial de las ecuaciones de Maxwell que emplean un segundo o cuarto orden de aproximación. En primer lugar, un monoestático UWB GPR se selecciona para evitar el acoplamiento entre la transmisión y las antenas de recepción. Esto elimina un importante artefacto de señal no deseada, que puede oscurecer la otra información en la señal. En segundo lugar, el transmisor ting/antena de recepción está situado sobre la superficie del GPR $[44,49]$. Además, el tamaño de los objetivos es mayor que la longitud de onda de la onda que se propaga GPR [45-50], (en las condiciones consideradas aquí). Por lo tanto, la dis persión de regreso objetivo debe ser significativa. En tales situaciones, el radar de ondas milimétricas puede proporcionar una solución alternativa y complementaria para las tareas de percepción [46-51].

El objetivo de este artículo es presentar el radar PELICAN, un radar de ondas milimétricas específicamente diseñado para aplicaciones de robótica móvil, que incluye la detección de obstáculos, la cartografía y la conciencia de la situación en general. En este primer trabajo de dos partes se explica la elección de un radar de ondas continuas modulado en frecuencia y se detallan los elementos teóricos de esta solución así: entre más microscópicas se observen las ondas en su comportamiento, mejor caracterización podemos tener de ellas. Esto aportaría algo importante a nuestra investigación, como trabajar patrones pequeños de ondas y con características relevantes para construir nuestro algoritmo y simulación, si es el caso. Además de las diferentes aplicaciones que se le han dado dentro de la geofísica [47-52], que ve la importancia del estudio de las ondas penetrantes por la información que traen para ser codificada y controlada $y$, de esta manera, obtener un mejor beneficio, como lo plantean los artículos que en este caso centran su estudio en las GPR [48-60], pero sin ver lo microscópico del asunto que aportaría mucha información a los modelos por construir.

\section{INTERPRETACIÓN DE LOS RESULTADOS}

Estimación de la permitividad dieléctrica, la atenuación y el contraste dieléctrico: para frecuencias que oscilan entre $0,8 \mathrm{GHz}$ y $2.8 \mathrm{GHz}$ y un rango intermedio de $1,4 \mathrm{GHz}$ para los dos tipos de suelo, el complejo permisividad dieléctrica relativa es aproximado. Diferentes fracciones de contenido de agua se seleccionan, que cubren entre el contenido residual de agua, $\theta r$, y el contenido de agua saturado, theta s (véase la Tabla 1). En la Figura 3 , los valores reales e imaginarios estimados de permitividad $(\epsilon)$ para el suelo franco limo y arena arcillosa se muestran como una función de la frecuencia (en contenido de agua $10 \%$ ), y como una función del contenido de agua (en $0,8 \mathrm{GHz}$ y $2,5 \mathrm{GHz}$ ).

Teniendo en cuenta el modelo empírico, diferentes valores de las partes real e imaginaria de $\varepsilon$ se encuentran en la Figura 3, determinados para ( $\mathrm{A}$ y $\mathrm{B}$ ) para frecuencias por debajo y por encima de $1,4 \mathrm{GHz}$; para ( $\mathrm{C}$ y D) para frecuencias por debajo y por encima de $0,8 \mathrm{GHz}$ : y para (E y F) para frecuencias por debajo y por encima de 2,5 $\mathrm{GHz}$. 

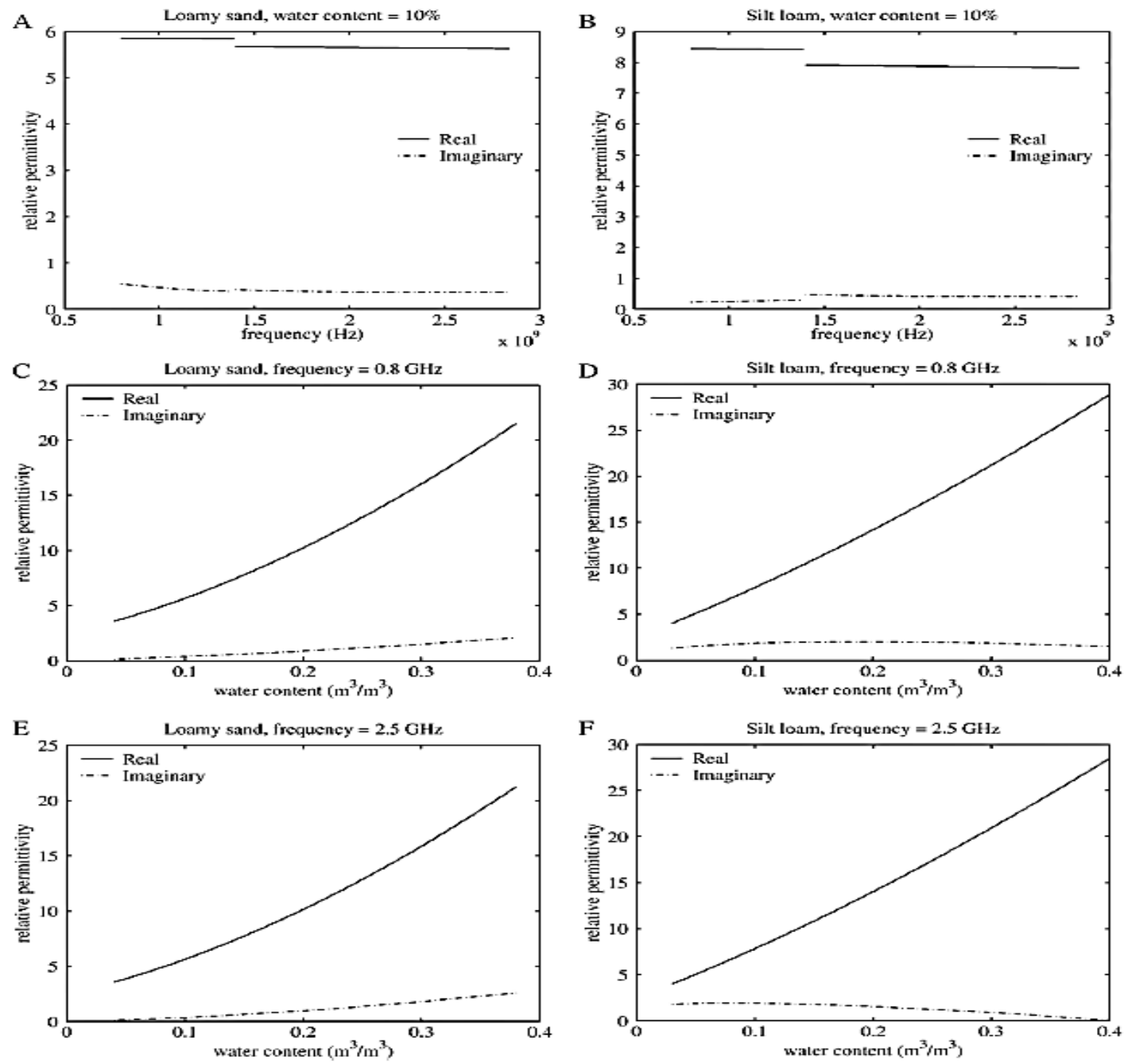

Figura 3. Ejemplos del complejo de permitividad dieléctrica relativa, calculada tanto para suelos como una función de: (A y B) de frecuencia, en $10 \%$ de contenido de agua, ( $C$ y D), a $0,8 \mathrm{GHz}$, en contenido de agua al $10 \%$ ( $\mathrm{E}$ y $\mathrm{F}$ ) también con la característica del $10 \%$ de agua, a $2,5 \mathrm{GHz}$, de frecuencia. Resultados del suelo franco limoso están en la columna de la izquierda y los de la tierra de arena arcillosa están en la columna de la derecha.

\section{CONCLUSIONES}

En revisión bibliográfica se pudo ver que existe un modelo EM, basado en funciones pedotransferencia, acoplado a un modelo 1D EM FDTD para predecir los efectos de las propiedades del suelo y de destino en el rendimiento de detección de GPR, con propiedades magnéticas del suelo y la textura del suelo, con las que se miden y se determinan previamente. Se incluyen en funciones pedotransferencia con el fin de estimar las propiedades eléctricas dependientes de la frecuencia de suelo. Estos últimos se utilizan para simular objeti- vos enterrados, con la señal retrodispersada utilizando el código de GprMax FDTD, para diferentes fracciones del contenido de agua y diferentes configuraciones de la altura de la antena y la profundidad del objetivo. El análisis de los resultados nos permite determinar la influencia de las propiedades electromagnéticas de los suelos, así como la de las características de destino, en el rendimiento de detección GPR.

La predicción de los efectos de las propiedades del suelo y de destino en el rendimiento de la detección del GPR se centra en dos suelos afectados por las minas 
de representación en Colombia. Se consideran diferentes fracciones tés de contenido de agua, así como las minas terrestres y artefactos explosivos AP locales. A partir de los valores de EM estimados, se calculan el factor de atenuación y el contraste dieléctrico. Los efectos de tales parámetros en la SNR son validados por las simulaciones. Aunque hay algunos factores que son omitidos en nuestro enfoque, tales como una superficie rugosa o el suelo heterogéneo, se puede predecir a partir de los resultados de escenarios favorables para la detección de minas terrestres utilizando un GPR monoestático en la banda de frecuencia de 0,8 a $2,8 \mathrm{GHz}$. Un sistema de este tipo, con un alto rango dinámico lineal, podría ser bastante adecuado para complementar la localización de minas antipersonas por los detectores de metales en los ambientes seleccionados.

La metodología que aquí se presenta se puede aplicar a otros tipos de suelo y los objetivos para predecir el rendimiento de detección de GPR (tenga en cuenta que, en este documento, nos referimos solo a la detección de objetos. Un proceso a posteriori tiene que llevarse a cabo para identificar y clasificar el objetivo detectado como un objeto de la mina o similar a un objeto de usar). Sin embargo, debe hacerse una comparación en laboratorio y mediciones en situaciones, con el fin de determinar la relevancia del error introducido por el hueco de la permitividad dieléctrica estimado, utilizando los modelos de Dobson et al. y Peplinski et al. El empleo de las redes neuronales nos permite ahorrar recursos de programación y procesamientos de máquina, ya que estas redes pueden compartir mucha información por varios caminos de comunicación que, al final, dejarán obtener la mayoría de los datos en el caso de un estudio de las ondas.

La tecnología ha mostrado diferentes formas de abordar el tema de detección de ondas por entrenamiento de redes neuronales, configuración de robots, como es el caso del Pelican, que buscan una mejor identificación del comportamiento de las señales que podrían ser implementadas en la detección de objetos enterrados, como en la investigación en relación con las minas antipersonales con los parámetros de comportamiento de la absorción de energía por el suelo, como factor influyente en las ondas que van a ser capturadas.

\section{REFERENCIAS}

[1] L.H. Morales-Pinto, M.C. Fuentes, J.A. Hernández. (2013, jul.). Monitoreo y control de vibraciones por efecto de voladuras en el túnel Sumapaz, concesión Bogotá Girardot. Revista Ingeniería, Investigación y Desarrollo. [En línea]. 13(2), 15-21. Disponible: http://revistas.uptc.edu.co/index.php/ingenieria_ sogamoso/article/view/3419

[2] O. Lopera y N. Milisavljevic. (2007). Prediction of the effects of soil and target properties on the antipersonnel landmine detection performance of ground-penetrating radar: A Colombian case study. Journal of Applied Geophysics. [En línea]. 63(1), 13-23.Disponible: https://doi.org/10.1016/j.jappgeo.2007.02.002

[3] F. Liu, Y. Ling, X. Xia y X. Shi. (2004). Wavelet methods for Ground Penetrating Radar imaging. Journal of Computational and Applied Mathematics. [En línea]. 169(2), 459-474. Disponible: https://doi. org/10.1016/j.cam.2003.12.036

[4] N.A. Duarte-Pinilla y H. Paz-Penagos. (2015, jul.). Justificación de una propuesta regulatoria para radiodifusión sonora y transmóviles en las fronteras colombianas. Revista Ingeniería, Investigación y Desarrollo. [En línea]. 15(2), 31-41. Disponible: http://revistas.uptc.edu.co/index.php/ingenieria_ sogamoso/article/view/4248

[5] A.J. Arrieta-Fuentes. (2016, jul.) Dispersión de material particulado (PM 10), con interrelación de factores meteorológicos y topográficos. Revista Ingeniería, Investigación y Desarrollo. [En línea]. 16(2), 43-54. Disponible: http://revistas.uptc.edu.co/index.php/ingenieria_sogamoso/article/view/5445

[6] C. Colla y C. Maierhofer. (2000). Investigations of historic masonry via radar reflection and tomography. Presentado en 2000 8th International Conference on Ground Penetrat- ing Radar (GPR). [En línea]. Disponible: http://proceedings.spiedigitallibrary.org/proceeding. aspx?articleid $=912896$

[7] S.A. Arcone y A.J. Delaney. (2000). Gpr images of hidden crevasses in Antarctica. Presentado en 2000 8th International Conference on Ground Penetrating Radar (GPR). [En línea]. Disponible: http://spie.org/Publications/Proceedings/Paper/10.1117/12.383512

[8] D. Daniels. (2004). Ground Penetrating Radar. Stevenage: IET Press. [En línea]. Disponible: http://digital-library.theiet.org/content/journals/10.1049/ el.2017.1570 
[9] C. van Coevorden, A. Bretones, M. Pantoja, F. Ruiz, S. García y R. Martin. (2006). Ga design of a thin-wire bow-tie antenna for $\mathrm{Gpr}$ applications. IEEE Trans. Geosci. Remote Sens. [En línea]. 44(4), 1004-1010. Disponible : http://ieeexplore.ieee. org/document/1610835/

[10] R.M. Morey, S.M. Conklin, S.P. Farrington y J.D. Shinnll. (1999). Tomographic Site Characterization Using CPT, ERT and GPR. [En línea]. Disponible: https://www.osti.gov/scitech/servlets/purl/773811

[11] N. Joachimowicz, C. Pichot y J.-P. Hugonin. (1991). Inverse scattering: an iterative numerical method for electromagnetic imaging. IEEE Trans. Antennas Propag. [En línea]. 39(12), 1742-1753. Disponible: http://ieeexplore.ieee.org/abstract/document/121595/

[12] C. Christodoulou y M. Georgiopoulos, Applications of Neural Networks in Electromagnetics. Norwood: Artech House, 2001.

[13] D. A. Gomes Vieira, Rede. perceptron com camadas paralelas (plp-parallel layer perceptron) (Ph.D. thesis), Universidade Federal de Minas Gerais, 2006.

[14] W.M. Caminhas, D.A.G. Vieira y J.A. Vasconcelos. (2003). Parallel layer perceptron, Neurocomputing. [En línea]. 55 (3-4), 771-778. Disponible: https:// www.researchgate.net/publication/222302650_ Parallel_layer_perceptron

[15] C. Christodoulou, J. Huang y M. Georgiopoulos. (1995). Design of gratings and frequency-selective surfaces using artmap neural networks, J. Electromagn. Waves Appl. [En línea]. 9 (1/2), 17-36. Disponible: http://tandfonline.com/doi/ abs/10.1163/156939395X00235?tab=permissions\&scroll=top\&

[16] M.K. Smail, Y.L. Bihan y L. Pichon, "Fast diagnosis of transmission lines using neural networks and principal component analysis", Int. J. Appl. Electromagn. Mech., vol. 39 n. 1, 2012, pp. 435-441.

[17] S. Caorsi y G. Cevini. (2005). An electromagnetic approach based on neural networks for the gpr investigation of buried cylinders, IEEE Geosci. Remote Sens. Lett. [En línea]. 2 (1) 3-7. Disponible: http:// ieeexplore.ieee.org/document/1381337/

[18] L. Newnham y A. Goodier. (2000). Using neural networks to interpret subsurface radar imagery of reinforced concrete 4084. [En línea]. Disponible: http:// proceedings.spiedigitallibrary.org/proceeding.aspx?articleid $=912595$

[19] X. Travassos, D. Vieira, N. Ida, C. Vollaire y A. Nicolas. (2008) Characterization of inclusions in a nonhomogeneous gpr problem by artificial neural networks, IEEE Trans. Magn. [En línea]. 44(6) 16301633. Disponible: http://ieeexplore.ieee.org/document/4527012/

[20] S.G. García, A.R. Bretones, B.G. Olmedo y R.G. Martn, "Finite difference time domain methods", in Time Domain Techniques in Computational Electromagnetics, D. Poljak (Ed.), Ashurst, UK: WIT Press, 2003, pp. 91-132.

[21] A. Giannopoulos. (1997). The investigation of transmission-line matrix and finite- difference time-domain methods for the forward problem of ground probing radar (Ph.D. thesis), University of York, 1997. [En línea]. Disponible: https://www. researchgate.net/publication/35719439_The_investigation_of_transmission-line_matrix_and_finite-difference_time-domain_methods_for_the_ forward_problem_of_ground_probing_radar

[22] R. Godoy-Rubio. (2005). Métodos de diferencias finitas incondicionalmente estables para la resolución de las ecuaciones de maxwell en el dominio del tiempo (Ph. D. thesis), Universidad de Granada. [En línea]. Disponible: https://dialnet.unirioja.es/servlet/tesis?codigo $=108732$

[23] B.G. Olmedo, S.G. García, A.R. Bretones y R.G. Martin. (2005). New trends in FDTD methods in computational electrodynamics: unconditionally stable schemes, in Recent Res. Development in Electronics, Transworld Research Network. [En línea]. Disponible en: http://personalpages.manchester. ac.uk/staff/fumie.costen/tmp/TRNEL18_corrected2.pdf

[24] R. Tibshirani, T. Hastie y J. Friedman, The Elements of Statistical Learning, New York City: Springer, 2001.

[25] S. Campana y S. Piro. (2008). Seeing the Unseen, Geophysics and Landscape Archae- ology. London: A Balkema Book, Taylor \& Francis. [En línea]. Disponible: http://www.crcnetbase.com/doi/ pdf/10.1201/9780203889558.fmatt

[26]J.B, Rodríguez, et al. (2015). A prediction algorithm for data analysis in GPR-based surveys. [En línea]. Disponible: https://www.researchgate.net/publication/279160326_A_Prediction_Algorithm_for_ Data_Analysis_in_GPR-based_Surveys

[27] S. Stergiopoulos. (2000). Advanced Signal Processing Handbook: Theory and Implementation for Radar, Sonar, and Medical Imaging Real Time Systems. Boca Raton: CR Press. [En línea]. Disponible: https://www.crcpress.com/Advanced-Signal-Processing-Handbook-Theory-and-Im- 
plementation-for-Radar/Stergiopoulos/p/ book/9781420037395

[28] L. Travassos, D.A.G. Vieira, N. Ida y A. Nicolas. (2009). In the use of parametric and non-parametric algorithms for the nondestructive evaluation of concrete struc- tures, Res. Nondestruct. Eval. [En línea]. 20(2), 71-93. Disponible: https://www.ncbi. nlm.nih.gov/pmc/articles/PMC3252010/

[29] F. Higuera, et al. (2013). Diseño y construcción de un prototipo de generador hidráulico para estudio y desarrollo de estrategias de control para la generación eléctrica en minas subterráneas, Revista Ingeniería, Investigación y Desarrollo. [En línea]. 13(2), 22-27. Disponible: http://revistas.uptc. edu.co/index.php/ingenieria_sogamoso/article/ view/3421

[30] D. A. Gomes Vieira, R. H. Caldeira Takahashi, V. Palade, J. A. Vasconcelos y W. Matos Caminhas, "The q -norm complexity measure and the minimum gradient method: a novel approach to the machine learning structural risk minimization problem", IEEE Trans. Neural Netw., vol. 19 n.ㅇ 8, 2008, pp. 1415-1430.

[31] I.T. Jolliffe. (2002). Principal Component Analysis. New York City: Springer. [En línea]. Disponible: http://www.springer.com/us/ book/9780387954424

[32] F. Rosenblatt. (1958). The perceptron: a probabilistic model for information storage and organization in the brain, Psychol. Rev. [En línea]. 65 (6), 386-408. Disponible: http://citeseerx.ist.psu.edu/ viewdoc/summary?doi=10.1.1.588.3775

[33] F. Rosenblatt, (1962). Principles of Neurodynamics. Washington:,Spartan Books. [En línea]. Disponible: https://catalog.hathitrust.org/Record/000203591

[34] M. Minsky y S. Papert, Principal Component Analysis. Cambridge, MA: MIT Press, 1969.

[35] H. Ortiz, I. Gómez, F. Angarita Cediel y C. A. Neira Triana. (2016). Amplificador operacional de transconductancia con alto rango modo común y bajo consumo de potencia. Ingeniería Investigación y Desarrollo [En línea]. 16(2), 78-83. Disponible: http://revistas.uptc.edu.co/index.php/ingenieria_ sogamoso/article/view/5448

[36] S. Grossberg. (1976). Adaptive pattern classification and universal recoding: I. parallel development and coding of neural feature detectors. Biol. Cybern. [En línea]. 23(3), 121-134. Disponible: https://link.springer.com/article/10.1007/ BF00344744
[37] T. Kohonen. (1972). Correlation matrix memories, IEEE Trans. Comput. [En línea]. C-21(4), 353-359. Disponible: $\quad \mathrm{http} / / /$ ieeexplore.ieee.org/document/5008975/

[38] K. Fukushima. (1980). Neocognitron: a self-organizing neural network model for a mechanism of pattern recognition unaffected by shift in position, Biol. Cybern. [En línea]. 36(4), 193-202. Disponible: https://www.ncbi.nlm.nih.gov/pubmed/7370364

[39] J.J. Hopfield. (2010). Neural networks and physical system with emergent collective computational abilities, Proc. Natl. Acad. Sci. [En línea]. 79, 2554-2558. Disponible: http://www.pnas.org/ content/79/8/2554.abstract

[40] D.E. Rumelhart, G.E. Hinton y R.J. Williams. (1986). Learning internal representations by error propagation, in: Parallel Distributed Processing: Explorations in the Microstructure of Cognition, vol. 1, Cambridge, MA, USA: MIT Press, pp. 318-362. [En línea]. Disponible: http://dl.acm.org/citation. cfm?id=104293

[41] J. Shing y R. Jang. (1993). Anfis: Adaptive-network-based fuzzy inference system, IEEE Trans. Syst. Man Cybern. [En línea]. 23, 665-685. Disponible: http://ieeexplore.ieee.org/document/256541/

[42] J.D. Toews, y W.Sirovyak. (2003). Metal Detector Trial - Colombia, DRDC Suffield TM 2003-099, pp. 3-7. [En línea]. Disponible: https://www.yumpu. com/en/document/view/16574172/metal-detector-trial-colombia-gichd

[43] Mullins, C.E. (1977). Magnetic susceptibility of the soil and its significance in soil science: a review, J. Soil Sci. [En línea]. 28, 223246. Disponible: http://onlinelibrary.wiley.com/ doi/10.1111/j.1365-2389.1977.tb02232.x/abstract

[44] J.A. Dearing. (1994). Environmental Magnetic Susceptibility. Kenilworth, UK: Chi Publishing. [En línea]. Disponible: http://onlinelibrary.wiley.com/ doi/10.1111/j.1365-246X.1996.tb04051.x/full

[45] A.S. Balanis, "Geometrical Theory of Diffraction" en Advanced Engineering Electromagnetics. New York: John Wiley \& Sons, 1989.

[46] M. Hagan y M.-B. Menhaj. (1994). Training feedforward networks with the Marquardt algorithm, IEEE Trans. Neural Netw. [En línea]. 5(6), 989-993. Disponible: http://ieeexplore.ieee.org/document/329697/

[47] C. Guattari, F. Amico y A. Benedetto. (2010). Integrated road pavement survey using gpr and Ifwd, in 2010 13th International Conference on Ground Penetrating Radar (GPR), 2010, pp. 1-6. [En línea]. 
Disponible: http://ieeexplore.ieee.org/xpl/mostRecentlssue.jsp?punumber $=5540532 \&$ filter\%3DAND\%28p_IS_Number\%3A5550063\%29\&pageNumber $=5$

[48] K.-Y. Huang y K.-S. Fu. (1987). Decision-theoretic approach for classification of ricker wavelets and detection of seismic anomalies, IEEE Trans. Geosci. Remote Sens. [En línea]. GE-25(2), 118-123. Disponible: http://ieeexplore.ieee.org/document/4072616/

[49] A. M Alani, M. Aboutalebi y G. Kilic. (2013). Applications of ground penetrating radar (GPR) in bridge deck monitoring and assessment. Journal of Applied Geophysics. [En línea]. 97, 45-54. Disponible: https://doi.org/10.1016/j.jappgeo.2013.04.009

[50] H. W. Yang, Z. Yang Kun y Y. Kun Pei. (2014). Ground-penetrating radar for soil and underground pipelines using the forward modeling simulation method. Optik International Journal for Light and Electron Optics. [En línea]. 125(23), 7075-7079. Disponible: https://doi.org/10.1016/j. ijleo.2014.08.099

[51] R. Rouveure, P. Faure y M.O. Monod. (2016). PELICAN: Panoramic millimeter-wave radarfor perception in mobile robotics applications, Part 1: Principles of FMCW radar and of 2D image construction. Robotics and Autonomous Systems. [En línea]. 81, 1-16. Disponible: https://doi.org/10.1016/j.robot.2016.04.001

[52] M. Rucka, J. Lachowicz y M. Zielioska, M. (2016). GPR investigation of the strengthening system of a historic masonry tower. Journal of Applied Geophysics. [En línea]. Disponible: https://doi. org/10.1016/j.jappgeo.2016.05.014

[53] A. Ruffell. (2006). Under-water Scene Investigation Using Ground Penetrating Radar (GPR) in the Search for a Sunken Jet ski, Northern Ireland. Science \& Justice. [En línea]. 46(4), 221-230. Disponible: https://www.ncbi.nlm.nih.gov/pubmed/17500424

[54] F. Sagnard, C. Norgeot, X. Derobert, V. Baltazart, E. Merliot, F. Derkx y B. Lebental. (2016). Utility detection and positioning on the urban site Sense-City using Ground-Penetrating Radar sys- tems. Measurement. [En línea]. 88, 318-330. Disponible: https://doi.org/10.1016/j.measurement.2016.03.044

[55] G.P. Tsoflias, S.L. De Vore y M. Lynott (2016). Combining ER and GPR surveys for evidence of prehistoric landscape construction: case study at Mound City, Ohio, USA. Journal of Applied Geophysics. [En línea]. 129, 178-186. Disponible: https://doi. org/10.1016/j.jappgeo.2016.04.002

[56] L. Seyfi y E. Yaldız. (2010). A novel software for an energy efficient GPR. Advances in Engineering Software. [En línea]. 41(10), 1195-1199. Disponible: http://dl.acm.org/citation.cfm?id=1862460

[57] D. Seyfried, R. Jansen y J. Schoebel (2014). Shielded loaded bowtie antenna incorporating the presence of paving structure for improved GPR pipe detection. Journal of Applied Geophysics. [En línea]. 111, 289-298. Disponible: https://doi.org/10.1016/j. jappgeo.2014.10.019

[58] D. Seyfried y J. Schoebel. (2015). Detection capability of a pulsed Ground Penetrating Radar utilizing an oscilloscope and Radargram Fusion Approach for optimal signal quality. Journal of Applied Geophysics. [En línea]. 118, 167-174. Disponible: https://doi.org/10.1016/j.jappgeo.2015.03.029

[59] D. Seyfried y J. Schoebel. (2016). Ground penetrating radar for asparagus detection. Journal of Applied Geophysics. [En línea]. 126, 191-197. Disponible: $\quad$ https://doi.org/10.1016/j.jappgeo.2016.01.022

[60] D. Seyfried, K. Schubert y J. Schoebel. (2014a). Investigations on the sensitivity of a stepped- frequency radar utilizing a vector network analyzer for Ground Penetrating Radar. Journal of Applied Geophysics. [En línea]. 111, 234-241. Disponible: http://www.sciencedirect.com/science/article/pii/ S0926985114003103

[61] A. Taflove y S. C. Hagness. (s.f.). Computational Electrodynamics: The Images of the front covers of Allen's FDTD books published in 1995. [En línea]. Disponible: http://www.ece.northwestern.edu/ ecefaculty/Allen1.html 